\title{
Application of Stable Distribution Theory-Based in Adaptive Filtering Algorithm
}

\author{
Qiudong $\mathrm{Yu}^{1, *}$ and Kari Olkkonen ${ }^{2}$ \\ ${ }^{I}$ Department of Computer and Information Engineering, Tianjin Agriculture University, Tianjin 300384, China \\ ${ }^{2}$ Department of Applied Physics, University of Eastern Finland, Kuopio, Finland
}

\begin{abstract}
In order to improve the performance of the adaptive Volterra filter, the tap length is often fixed in the study, but in practice, the values of the tap length is difficult to fix, which value is too large or too small are unable to meet the system requirements. This requires the fixed tap length becomes variable tap length during the study of the filter. This paper describes $\alpha$ stable distribution theory and the adaptive filter theory and introduces the lattice-type filter to get adaptive filter based on lattice filter. And this paper further improved on the basis of this algorithm, discussed the adaptive update algorithm of LSVP tap length of the algorithm and designed variable obtained taps LSVP algorithms. Finally, we get an adaptive channel equalization algorithm used in the simulation experiments. The results showed that the use of variable-tap LSVP algorithm can converge to the optimal length of the tap with more applicability.
\end{abstract}

Keywords: A stable distribution, variable tap length, adaptive filtering, LMP, LVSP.

\section{INTRODUCTION}

As science advances, more and more technical fields of engineering problems related to signal processing, signal is the carrier and the physical manifestation of information, the purpose of the signal processing is expected from the underlying data in noisy obtained by de-noising the desired information. The second-order statistics can be carried out take the Gaussian model during the process, but in practice, most of the signal types are incompatible with the Gaussian distribution, so we need to find a more reasonable signal model. This model is the $\alpha$ stable distribution model, $\alpha$ stable distribution is a generalized form of Gaussian distribution, which can maintain consistent with the actual data, the model is more suitable to describe some random signals.

The concept of $\alpha$ stable distribution was first proposed from French mathematician Levy; he found that in the central limit theorem in 1925, when a limited relaxation of the conditions of the variance, subject to the limit distribution is stable distribution. Then he studied the stable distribution using the infinitely divisible distributions based on the stable distribution Fourier transform form. 1939 Doblin putted forward modern stable distribution rules. After tireless research of many late mathematicians $\alpha$ stable distribution, $\alpha$ stable distribution has already formed a relatively complete theoretical system currently. But until 1993, a stable distribution get attention and application in the field of signal processing, and in terms of adaptive filtering, $\alpha$ stable distribution application signal theory has been successfully applied. Wherein the LMP and LMAD algorithm are proposed based on minimum dispersion criterion; NLMP algorithm based on the LMP algorithm can improve the stability and the convergence speed of the algorithm; further promote NLMP algorithm and then mixing the mixed smallest average norm algorithm can form a mixture of toughness minimum average norm algorithm. In the domestic study, $\alpha$ stable distribution of adaptive filtering theory algorithm is also very active, not only to further the study of algorithms, as well as new algorithms inspired by the previous algorithms proposed.

In statistical signal and adaptive signal processing, the most important is the adaptive filter theory, adaptive filter has a wide range of applications in many fields, because adaptive filter without prior knowledge, it only needs to meet the criteria to achieve optimal performance of the filter under the circumstances, the adaptive filter only needs to adapt its parameters on various criteria in the process. From the current direction of development, the main directions of development of adaptive filter are the depth research on $\alpha$ stable distribution.

A typical composition of the adaptive filter is basically same, the structure shown in Fig. (1), and the ultimate goal of the adaptive filter are looking for the best coefficients, but in reality, its shape is variable. The core of adaptive filter algorithm is adaptive filtering, adaptive algorithm now has four branches, each branch of study which also has the appearance of an endless stream of new algorithms, and each algorithm is derived in order to get the most gifted programs of the typical problems with research value and significance.

Based on the second order Volterra filter and variable length tap of adaptive algorithm, this paper study the LMP adaptive filtering algorithm. This algorithm first simplify the 


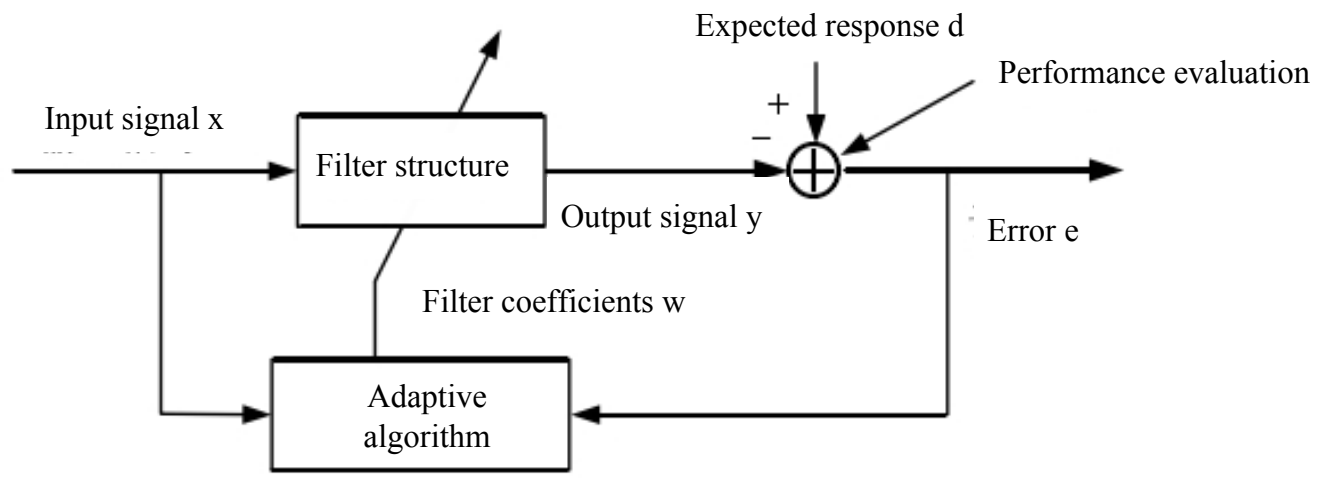

Fig. (1). Composed of an adaptive filter.

filter structure, operation method is to process the input signal of filter, reduce weight coefficient quadratic term in order to achieve the second-type signal decoupling, so the tap length line type and nonlinear parts are identical. After simplifying the structure of the filter, the adaptive of filter tap length is the next.

\section{A STABLE DISTRIBUTION}

$\alpha$ stable distribution is the generalized Gaussian distribution with a wide range of applicability, but the random statistic signal of $\alpha$ stable distribution is low-level, on this basis, we introduced the definition, the nature and its probability density function of $\alpha$ stable distribution.

\subsection{Definition and Properties}

From different angles can give different definitions ways to $\alpha$ stable distribution, according to the stability definition, we can see that regardless of the addition or the probability density of the convolution of random variables are closed, and the stable distribution and linear combinations are subject to stable distribution, and parameter values are the same, where $\alpha$ is called stability factor. According to its definition domain of attraction, it can also know as the generalized central limit theory, which is a statement from the central limit theory perspective. According to the definition of its characteristic function, it is the definition from $\alpha$ stable distribution characteristic function on the basis of the existence of a uniform. The characteristic function is determined by the standard parameters of the system, the system parameter by the standard four parameters, namely the characteristic index determines the thickness of the probability density function; skew parameter determine the degree of symmetry of the distribution; scale parameter determine the sample bias decision metric and position parameter indicates the mean or median value.

$\alpha$ stable distribution has nine kinds of basic nature, this article does not enumerate the content of the basic nature.

These basic properties were given in exchange for a stable distribution, symmetrical, and can indicate the scale and displacement distribution is stable distribution theoretical basis of low-level statistics.

\subsection{Probability Density Function}

Since the probability density function of $\alpha$ stable distribution does not have the parse form, depending on the characteristics and application areas of $\alpha$ stable distribution, it can be used to calculate the probability density function in different ways.

$\alpha$ stable distribution has a characteristic that its characteristic function and the probability density function are the Fourier transform of each other. According to this feature, we can take the inverse transform characteristic function to calculate the probability density function:

$$
f(x ; \alpha, \beta)=\frac{1}{\pi} \int_{0}^{\infty} \exp \left(-t^{\alpha}\right) \cos \left[x t+\beta t^{\alpha} \omega(t, \alpha)\right] d t
$$

From the formula (1), the probability density function of $\alpha$ stable distribution is continuous, the reciprocal of any order are also exist, but the probability density function of the applicability of this method was not strong, because the calculation of the amount is too large.

We also can by a probability density function of $\alpha$ stable distribution to power series expansion to get its analytical solution, after expansion, it becomes the following forms, and we can simplify it to the equation (3):

$$
\begin{aligned}
& f(x ; \alpha, \beta)=\left\{\begin{array}{l}
\frac{1}{\pi x} \sum_{k-1}^{\infty} \frac{(-1)^{k-1}}{k !} \Gamma(\alpha k+1)\left(\frac{x}{r}\right)^{-\alpha k} \sin \left(\frac{k \pi}{2}(\alpha+\xi)\right), 0<\alpha<1 \\
\frac{1}{\pi x} \sum_{k-1}^{\infty} \frac{(-1)^{k-1}}{k !} \Gamma\left(\frac{k}{\alpha}+1\right)\left(\frac{x}{r}\right)^{k} \sin \left(\frac{k \pi}{2 \alpha}(\alpha+\xi)\right), 1<\alpha \leq 2
\end{array}\right. \\
& f(x ; \alpha)=\left\{\begin{array}{l}
\frac{1}{\pi x} \sum_{k-1}^{\infty} \frac{(-1)^{k-1}}{k !} \Gamma(\alpha k+1)(x)^{-\alpha k} \sin \left(\frac{k \pi \alpha}{2}\right), 0<\alpha<1 \\
\frac{1}{\pi\left(x^{2}+1\right)}, \quad \alpha=1 \\
\frac{1}{\pi x} \sum_{k-1}^{\infty} \frac{(-1)^{k}}{2 k !} \Gamma\left(\frac{2 k+1}{\alpha}\right)(x)^{2 k}, 1<\alpha \leq 2 \\
\frac{1}{2 \sqrt{\pi}} \exp \left(\frac{x^{2}}{4}\right), \quad \alpha=2
\end{array}\right.
\end{aligned}
$$


Calculation methods of power series expansion method are approximate, and the results are often not accurate enough, but this method does not take into account all the distribution range of samples, now only apply in extreme cases: larger or smaller argument.

Currently, there is a way to make up for the lack of the above two methods, not only to get the analytical formula, but a smaller amount of calculation. This method is the finite Gaussian hybrid approximation based on the mixing characteristics of random variable. Analytical expression for the probability density function is as follows:

$P_{\alpha, 0, \gamma, \delta}(z)=\frac{\sum_{i=1}^{N} 2 \exp \left(-\frac{(z-\delta)^{2}}{2 \gamma v_{i}^{2}} f_{Y}\left(v_{i}^{2}\right)\right)}{\sum_{i=1}^{N} f_{Y}\left(v_{i}^{2}\right)}$

\section{THE SECOND ORDER VOLTERRA ADAPTIVE FILTERING ALGORITHM OF VARIABLE TAP LENGTH}

\subsection{Second order Volterra LMP Algorithm}

Under normal circumstances, the second order Volterra filter input and output can be used to represent in Equation 5:

$$
y(n)=\sum_{i=1}^{N-1} h_{i}(n) x(n-i)+\sum_{i=0}^{N-1} \sum_{j=0}^{N-1} h_{i, j}(n) x(n-i) x(n-j)
$$

Wherein the linear and non-linear portion coefficients of the filter are given in the formula, in accordance with the symmetry of the filter core assumption, Equation 4 may be expressed by the following equation:

$$
y(n)=H_{1}^{T} X+X^{T} H_{2} X
$$

Where:

$$
\begin{aligned}
& X=[x(n), x(n-1), \ldots, x(n-N+1)] T \\
& H_{1}=\left[h_{0}(n), h_{1}(n), \ldots, h_{N-1}(n)\right]^{T}
\end{aligned}
$$

$$
H_{2}=\left[\begin{array}{llll}
h_{0.0} & h_{0.1} & \cdots & h_{0 . N-1} \\
h_{1.0} & h_{1.1} & \cdots & h_{1 . N-1} \\
\cdots & \cdots & \cdots & \cdots \\
h_{N-1.0} & h_{N-1.1} & \cdots & h_{N-1 . N-1}
\end{array}\right]
$$

As mentioned above, the premise of re-expression is the nuclear symmetry of the filter, so we can introduce a matrix $\mathrm{Q}$, the matrix is composed by eigenvectors $\mathrm{H} 2, \mathrm{H} 2$ can symmetric matrix diagonalization, the keratosis $\mathrm{H} 2$ into equation 6 , you can get a more simplified expression filter input-output relationship of the formula:

$$
y(n)=H_{3}^{T} X_{1}+H_{4}^{T} X_{2}
$$

However, after the introduction of the matrix Q, the orthogonal changes meet their requirements have a lot, after comparison, we found a quadrature of the input signal can be filtered adaptive, such filtering has better orthogonal compared with other orthogonal transformation, this filtering is the lattice type filter. The paper selected level N-1 lattice filter. The introduction of this filter can realize the quadrature of the input signal from the filter, thereby converting the input signal into the prediction error sequence. From the above expressions, we can draw the filter structure based on the adaptive lattice filter, the tap length of the linear portion and non-linear portion of the structure are same, the adaptive weight factor is $2 \mathrm{~N}$, and the output signal is a linear function of the weight coefficient. It is worth noting that, in the Volterra LMP adaptive filtering algorithm based on lattice second order designed in this paper (referred to as LSVP algorithm), the weights are to be adjusted, the adjustment formula was not listed in this article, but in the adjustment formula, the selecting of formula parameters should ensure a premise, that is, to ensure that the adjustment algorithm is convergent.

\subsection{Adaptive Control Algorithm of Variable Length Taps}

In previous studies of the Volterra filter, in order to improve the performance of the adaptive Volterra filter, the tap length is often fixed. But in practice, the length of the value of the tap is difficult to fix, is too large or too small values are not satisfy system requirements, which requires the length of the fixed tap into a variable length of the filter in the study. In the above study of LSVP algorithm, the tap length is fixed, so we have to improve on its basis, further discussion of adaptive update algorithm for tap length. Because the tap length good performance when it is long, but increase the amount of computation and error values; tap length is short with fast convergence, but cannot meet the performance requirements, so we should take comprehensive consideration of the above advantages and disadvantages, make the judge of tap length and try to play the advantages with different tap length when we changed the tap length during design. As to determine the length of the tap, we have adopted the standard method. In the filter update process, we have to determine the actual situation at this time whether the tap length play advantage, when the tap is too long, you can make the tap length of filter shorter; hand, when the tap length is too short, put the tap length of the filter becomes long. Then under the $\alpha$ stable distribution noise background, we can determine the optimal length of taps by the following ways: First, we assume that the filter tap length is L, p-order steady-state error can be determined after setting the length, we have to determine this error minimum or infinitely close to the minimum, the corresponds tap length is considered to be the most tap length. In this article, in order to the best estimate for a more flexible tap length, we introduce the concept of fractional tap-length, first we get the p-order estimate steady-state error by recursively, and then get the optimal length by iterative calculation of the tap-tap-length score. Equation 11 is the fractional tap-length iterative process: 


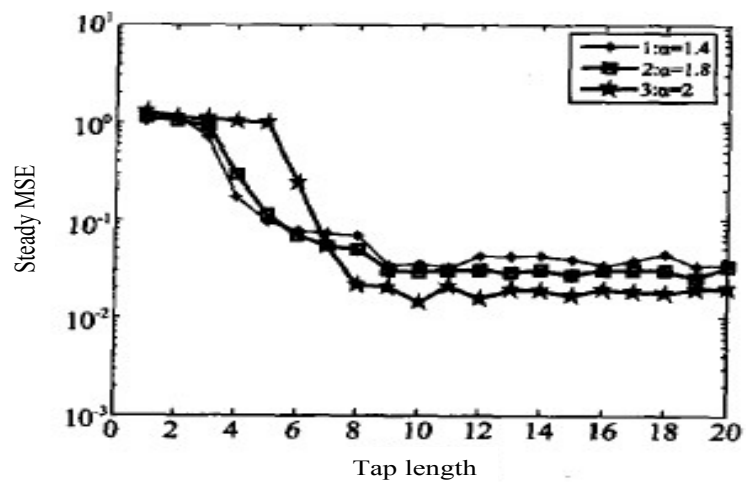

Fig. (2). Diagram of the tap length and steady learning curve under different characteristics index case.

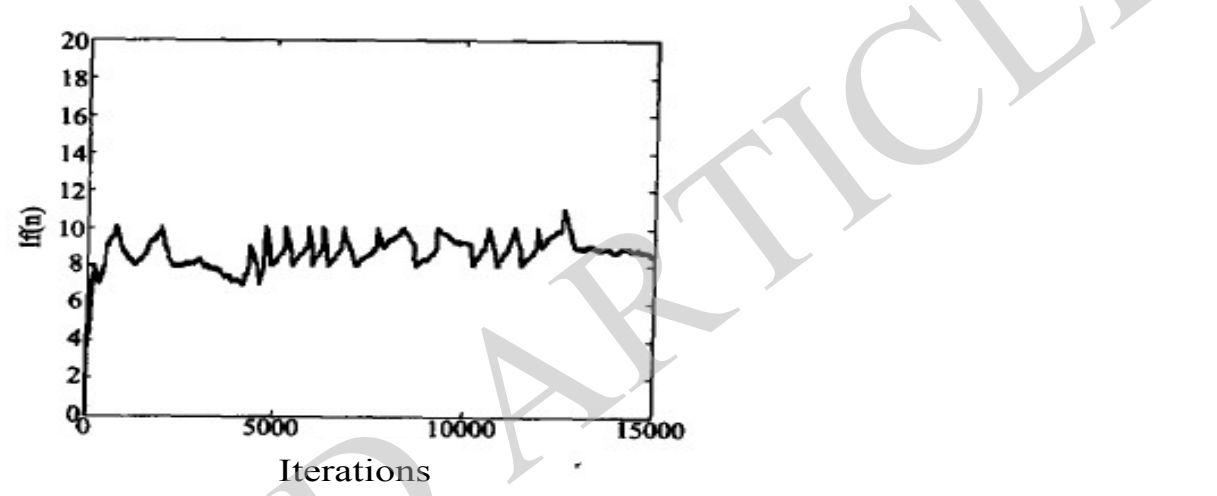

Fig. (3). Convergence of tap length scores with characterized index is 1.8, the initial length of tap is 4 .

$l_{f}(n+1)=\left(l_{f}(n)-\eta\right)+\beta \theta(\xi(n-1)-\xi(n))$

Integer of the fraction length by iterations is the true tap length we want. In the formula, nand $\beta$ are the threshold value we set, which determines the length of the tap need to increase or decrease, it is worth noting that the threshold values should be properly and cannot be too large or too small, too large nwill cause the offset error and lead to the optimal value of the length of the tap is not accurate; too large $\beta$ may cause the calculation process becomes complicated, so that the filter cannot converge to the optimum weights. According to update the weight coefficients, p-order steadystate error and updates of the tap length, the LSVP algorithm based on the above variable tap length has been designed to complete.

\section{SIMULATION}

In order to verify whether the completed algorithm designed can meet the actual needs; we take the variable tap LSVP algorithm to apply in the analysis of the practical performance of the algorithm. In the following, we apply the algorithm to adaptive channel equalization, which, $H(z)=J(z)+0.2 J^{2}(z)$ is the channel transfer function, the threshold $\eta$ and $\beta$ are respectively 0.1 and 0.004 , $J(z)=0.3842+0.8704 z^{-1}+0.3842 z^{-2}$. The experiments were carried out 30 times, and finally the resulting curve is the average of the results of 30 times.

Assumed the SNR is $30 \mathrm{~dB}$, respectively, the performance of the algorithm under the two background noise are calculated, noise background were chosen are $\alpha$ stable distribution noise and Gaussian noise. Where $\alpha$ stable distribution, we set the dispersion coefficient is 1 , symmetrical parameter and unknown parameter under two noise backgrounds are 0 , and the feature coefficients are set to $1.4,1.8$ and 2. First, we give different characteristics index case, the diagram of length of the tap and the steady learning curve Fig. (3). The figure shows that under different feature indices, there are different optimal tap lengths. When the characteristic index is less than 2, the optimal tap length is 9; when the characteristic exponent equal to 2 , the optimal length has been reduced to 8 .

Under the $\alpha$ stable distribution context, selected feature index of 1.8, when comparing the length of the convergence of different initial tap-tap-length score, initial tap length we were selected as 4 and 16, Figs. (3) and (4) show that when the initial tap length of 4 , the convergence of tap-length score; Figs. (3) and (4) shows the tap when the initial length of 16 , the convergence of tap-length score. By comparing the two figures, no matter the initial tap length is larger or smaller than the optimal length, by varying tap this article LSVP algorithm can eventually converge to 9 , which is the best tap 


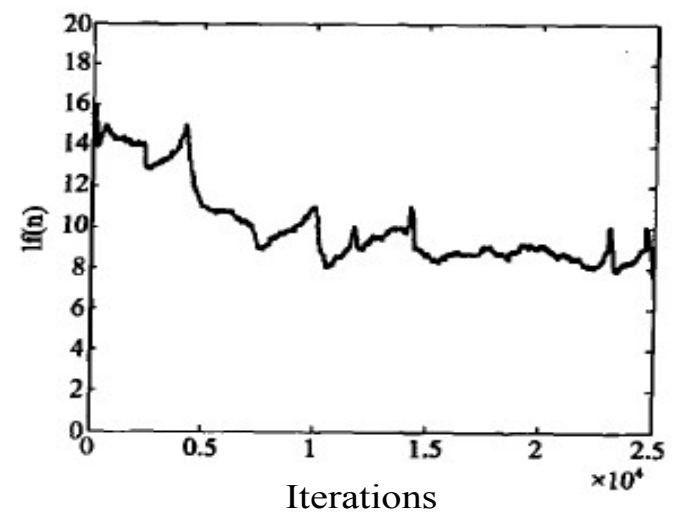

Fig. (4). Convergence of tap length scores with characterized index is 1.8 , the initial length of tap is 16.

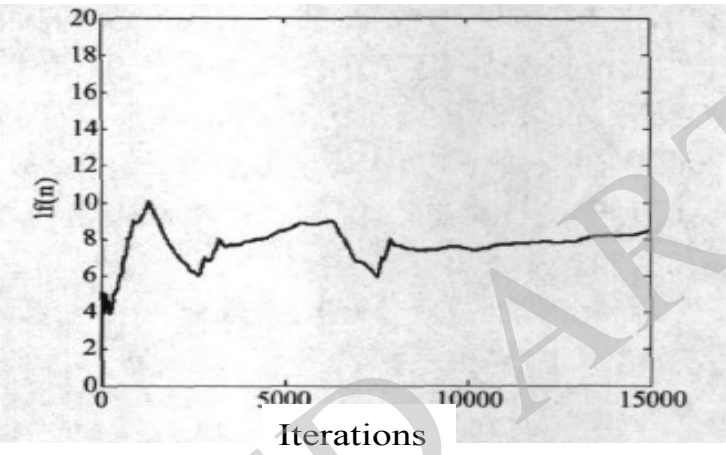

Fig. (5). Convergence of tap length scores with characterized index is 2, the initial length of tap is 4 .

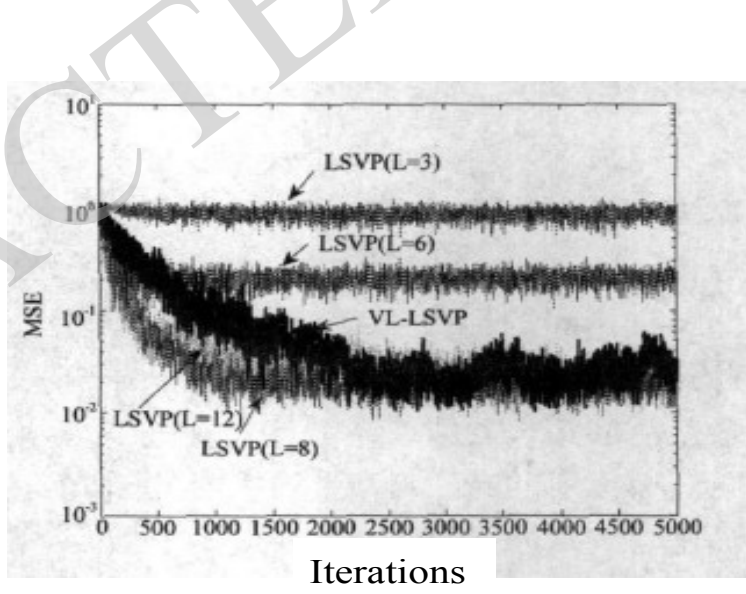

Fig. (6). Learning curve diagram of two algorithms with characterized index is 2 .

length. But it is worth note that, when the initial tap length shorter than the optimum length of the tap, the tap length of the fraction of relatively fast convergence.

Seen from the foregoing, in the context of Gaussian noise, that is the characteristic index of 2, the optimal tap length should be 8 . Under this premise, we given the convergence of the initial tap length scores was 4 and the learning curve of the two algorithms in Figs. (5) and (6). Fig. (5) shows that, in the context of Gaussian noise, although the initial tap length is less than optimal tap length, but in the end it still converge to the optimal tap length. Fig. (6) shows, when the selected tap length is less than optimal tap length, variable tap LSVP algorithm is superior to LSVP algorithm, the error is relatively small; when the tap length is greater than the optimal length tap, the two algorithms have the same degree of convergence, but obviously the extent of the fixed taps obviously complicated calculation algorithm. From the above analysis, the variable tap LSVP algorithm designed not only to get the optimal tap length, while a strong stability. 


\section{CONCLUSION}

This article describes $\alpha$ stable distribution theory and adaptive filter theory, and introduced lattice-type filter on the basis of second order Volterra filter. The introduction of this filter can realize orthogonal change of the filter input signal, thereby converted the input signal into a sequence of the prediction error, obtained adaptive filter based on lattice-type filter. But above lattice filter based adaptive filter, which tap length is fixed, we have further improved on the basis of this algorithm, discussed the adaptive update algorithm of the tap length of LSVP algorithm to design derived variable tap LSVP algorithms. Finally, we put the algorithm we get into the adaptive channel equalization to take the simulation experiments. The results show that, even if the different characteristics of the index, which is the background noise is not the same, the use of variable-tap LSVP algorithm can converge to the optimal length of the tap, and improved algorithms had more applicability relatively, which can better meet the actual work needs.

\section{CONFLICT OF INTEREST}

The author confirms that this article content has no conflict of interest.

\section{ACKNOWLEDGEMENTS}

2013 Tianjin Innovation Fund Project (No.13ZXCX GX09200): Pulse high precision trajectory real-time positioning system.
2014 Tianjin Agricultural Science and Technology Achievements Transformation and Popularization Project (No.201404030): The integration and technical demonstration of animal husbandry intelligent precision farming on internet of things.

2012 Tianjin Innovation Fund Project (No.12C2 6211200327): The aid-system for special education for disabled persons on embedded video technology.

\section{REFERENCES}

[1] Z. F. Ye, Statistical Signal Processing. Anhui: China University of Science and Technology Press, 2009.

[2] Z. J. Lv and X. C. Xiao, "A new adaptive beam forming technology", Systems Engineering and Electronics, yol. 25, no.5, pp. 542-545, 2003.

[3] C. Lin and Q. Y. Feng, "Adaptive filter algorithm of variable tap length LMS", Electronics \& Information Technology, vol. 30, no. 7, pp. 1676-1679, 2008.

[4] Z. L. Liu, "The Dynamic Analysis of China's Energy-EconomyEnvironment System: VAR and VEC Modeling", Advances in Information Sciences and Service Sciences, vol. 4, no. 14, pp. 210$218,2012$.

[5] Y. P. Zhang, N. N. Zheng, and C. H. Li, "Adaptive volterra filter design of image edge extraction", Journal of Electronics, vol. 27, no. 4, pp. 75-78, 1997.

[6] C. -C. Chen, "A FMEA-aided Project Bidding Decision System", Advances in Industrial Engineering and Management, vol. 3, no. 1, pp. 21-28, 2014.

[7] Y. H. Lei, C. Y. Song, and S. Q. Ding, "Nonlinear prediction of chaotic water based on volterra adaptive filter", Harbin Engineering University, vol. 28, no. 10, pp. 1127-1130, 2007

\footnotetext{
Received: September 16, $2014 \quad$ Revised: December 23, $2014 \quad$ Accepted: December 31, 2014

(C) Yu and Olkkonen; Licensee Bentham Open.

This is an open access article licensed under the terms of the Creative Commons Attribution Non-Commercial License (http://creativecommons.org/licenses/by-nc/3.0/) which permits unrestricted, non-commercial use, distribution and reproduction in any medium, provided the work is properly cited.
} 